\title{
Optical Device Can Measure the Glass Properties
}

\author{
Kamyar Karimi Fakhr \\ Department of Physics, University of Melbourne (Trinity College), Melbourne, Victoria 3000, Australia
}

\begin{abstract}
We will discuss how you can measure the glass properties in light of thickness and refractive index with a portable and small device and how it works based on an optical theory. The main industries that you can use it are air industry (for airplanes windows and building and construction). Thus, the probe into glass properties is very meaningful with various practical applications.
\end{abstract}

Key words: Optics, glass, laser, light beams.

\section{Introduction}

An optical device has been constructed to determine the properties of a glass sheet such as refractive index and thickness without direct contact. One of the standard methods to determine the refractive index is semicircle shaped glass. But here it is a method to overcome the disadvantages of the previous devices and to provide thickness measuring apparatus which is able to determine the thickness of a transparent workplace in a high degree of accuracy and more inexpensively, due to its construction.

\section{Methodology and Experiment}

Laser light is impinged on the glass to provide a pair of beams reflected from the front and back surfaces of the glass plate respectively. These two points on the front of the device are detected by Pixycam which takes successive photos. These photos are sent to Arduino board in which by using different theories in Matlab. And the characteristics of glass can be obtained programmatically. Then these data are shown on an LED screen. Several glasses have been applied to find the results of this setup successfully.

\subsection{Glass}

This definition of nonmetallic materials is too general according to different standard, and taking

Corresponding author: Kamyar Karimi Fakhr, bachelor, research field: physics. silica gel for example, it would be a glass. Numerous other definitions tend to place most emphasis on viscosity behavior.

Glass is restricted to inorganic products, which in this general form is questionable. Until now glass has been considered essentially as a frozen-in supercooled liquid, and the possibilities for investigating solid bodies can be used to obtain information about this structure and its properties. The glass is not found in thermodynamic equilibrium, thermodynamic data can be ascertained and corresponding reflection from this can be made. One of the fundamental equations of thermodynamics has already been pointed out:

$$
G=H-T S
$$

where, $\mathrm{G}$ is the free enthalpy, $\mathrm{H}$ is the enthalpy or heat content. $\mathrm{T}$ is the absolute temperature, and $\mathrm{S}$ is entropy.

\subsection{Performance}

The below image shows the performance of such device that includes Pixycam, Arduino, laser pointer, simple ramp and a lens.

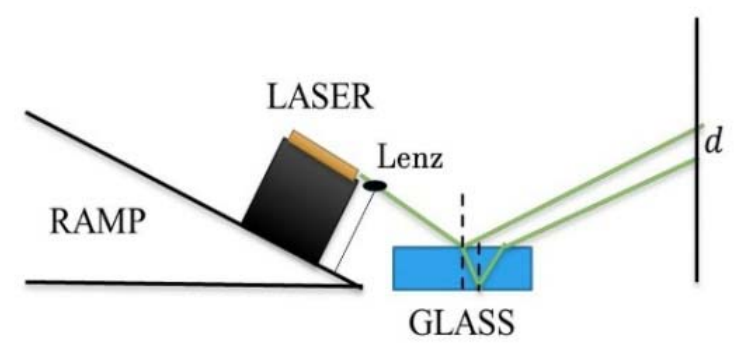


When the laser is one, light beams go through the lens, then minimize and reflect from the surfaces of the glass, after that pixy detect them and calculation process start with the Arduino.

\section{How to Measure}

The measurement of glass thickness could be simple. You can use ruler. If you want to be more accurate, you can conduct this measurement in the coliseum, which depends on your decision. And your position is related to glass properties. If you do not have enough time, ruler is fine just for thickness. The ray of light could be a best way to measure the glass properties because of its precision and speed. Laser beam is the answer for above question due to its slim ray and the power of gathering.

\subsection{Coherence of Light}

The classical theory of light describe optical phenomena is terms of electromagnetic oscillations. One of the basic tools of this theory is harmonic analysis. The variation of the electromagnetic field at a point is represented as the superposition of harmonic oscillation of the form:

$$
E=E_{I} \operatorname{CoS}\left(2 \pi V_{I} t-\varphi_{i}\right)
$$

where, $E_{I}$ is definite amplitude, $v_{i}$ is frequency and $\varphi_{i}$ is phase. The phase varies in space from point to point in a linear manner.

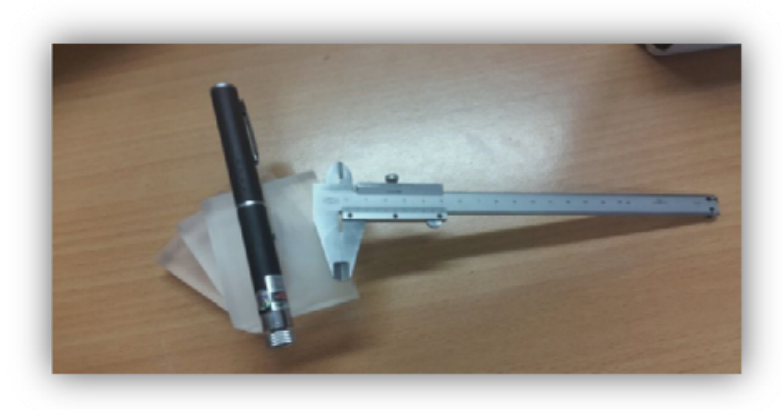

\subsection{Tools}

Basic tools of this measurement are: coliseum, small part of glasses and laser pointer (green recommended). These tools are for primary tests not for main tests!

\section{Programming}

This programming consists of two main parts: theoretical and practical. MATLAB does the theoretical and Arduino does the practical.

The most of the mathematical theory is trigonometric, from lamberts and snell's law to some complicated formula which converted to $\mathrm{C}++$ language and put into MATLAB software to earn the value of properties. All of those codes go through Arduino software. The most of code line is about founding the center of the dots in front of the pixycam, then find the distance between them and send the result to Arduino to realize the thickness with trigonometric. Threshold can help pixy to do that work and make the process easy for Arduino.

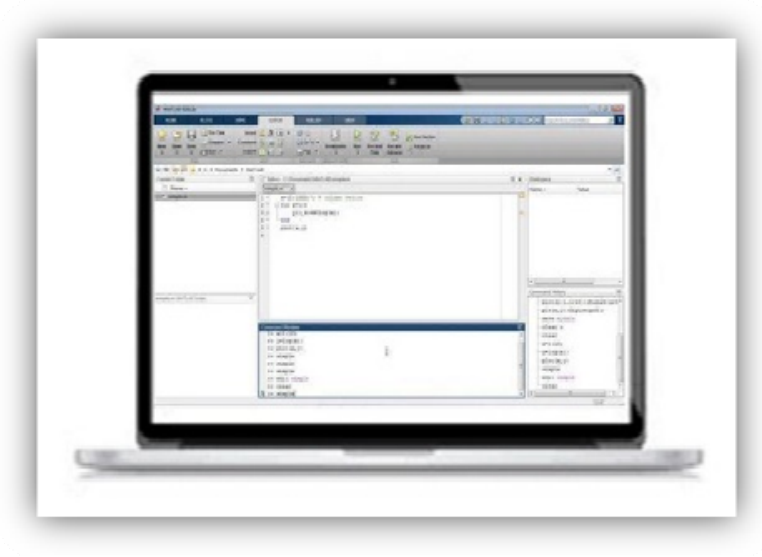

\section{Thickness chart}

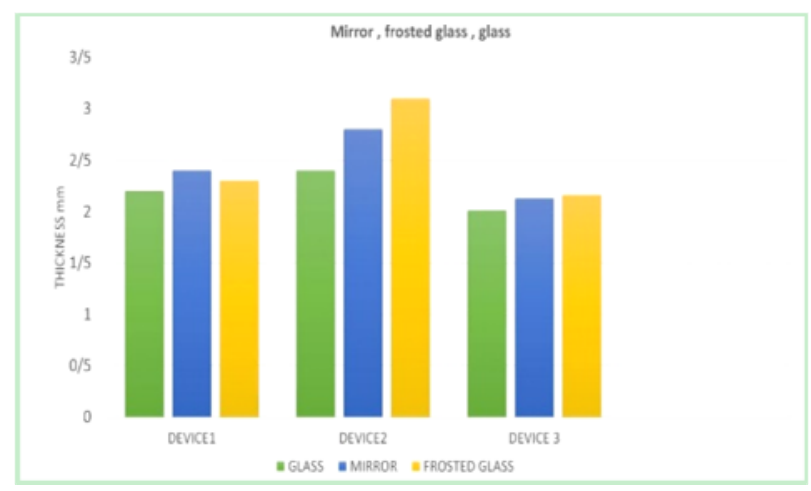

\section{Devices}

We did all tests with three types of pixy and Arduino to recognize the smallest errors and find out the best model of them for our device. (The chart shows the result of three devices which is the thickness of glasses). 


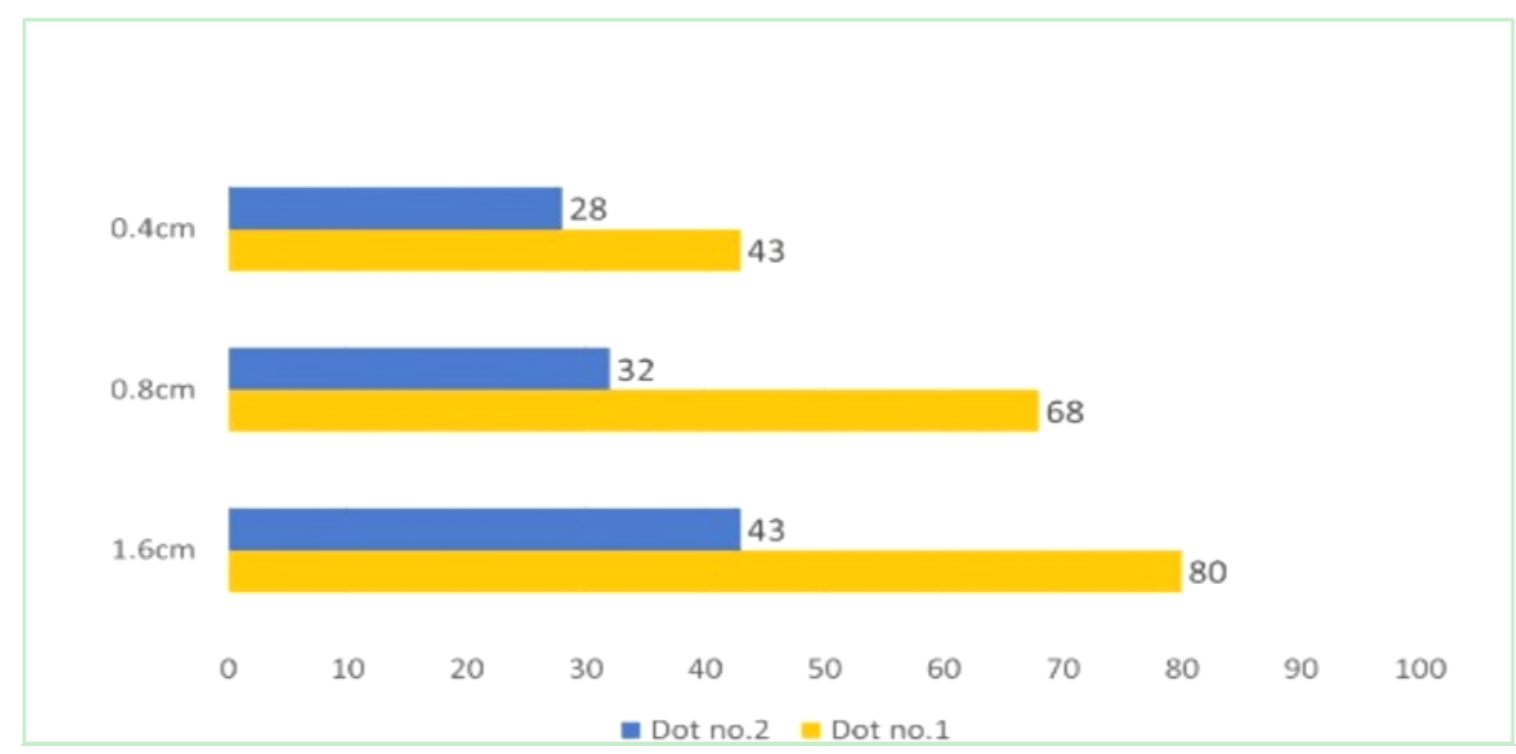

\section{The absorption of glass}

In this test we found the amount of absorption from dots that display in front of pixycam, they helped to make sure about the power of laser beam (Caution: the sum of dots absorption is not equal to $100 \%$ ) [1].

\section{Transparency}

$65 \%$

The transparency of our tested glass, we use low transparency to measure the accuracy of the device.

$20 \%$

Transparency can be reduced to about $20 \%$ because of dirtiness.

\section{Different Glasses}

The main subject which made by glasses are below materials:

- Frosted glass

- Mirror

- Soda glass

Frosted glasses are similar to normal glass but they are not transparent like glass and you cannot see the other side of them. Light raw cannot pass them completely due to their high refractive index, optical device use the powerful laser pointer and it can pass them and make another reflection from another side of them, then you can see two points in front of the pixycam.

Mirror is very familiar to us and most of us use it daily. Being unique, they reflect all of the light beams, have a high density and make a lot of point for pixycam detection. Because of that, we can use the power of spread method on MATLAB programming to separate the main reflection dots from other. In this method pixy can find the main dots just from the absorption amount as it shows on the above bar chart. Nowadays, there is no one who never drinks a cola or beer with the soda glass on their hands. Their manufacture formula is simpler than that of high quality and transparent glass. However, companies do not change their formation at all, but just take some manipulation on their silicon [2].

\subsection{Density}

Even if the density of glass does not have great practical importance for its usage, it is appropriate for several reasons to discuss it as the next property, for its values are often needed for calculation of other properties [3]. In addition the constancy of composition of a glass can be checked in a simple way with density measurement. For purposes of definition, the density $\rho$ is the mass per unit volume. 


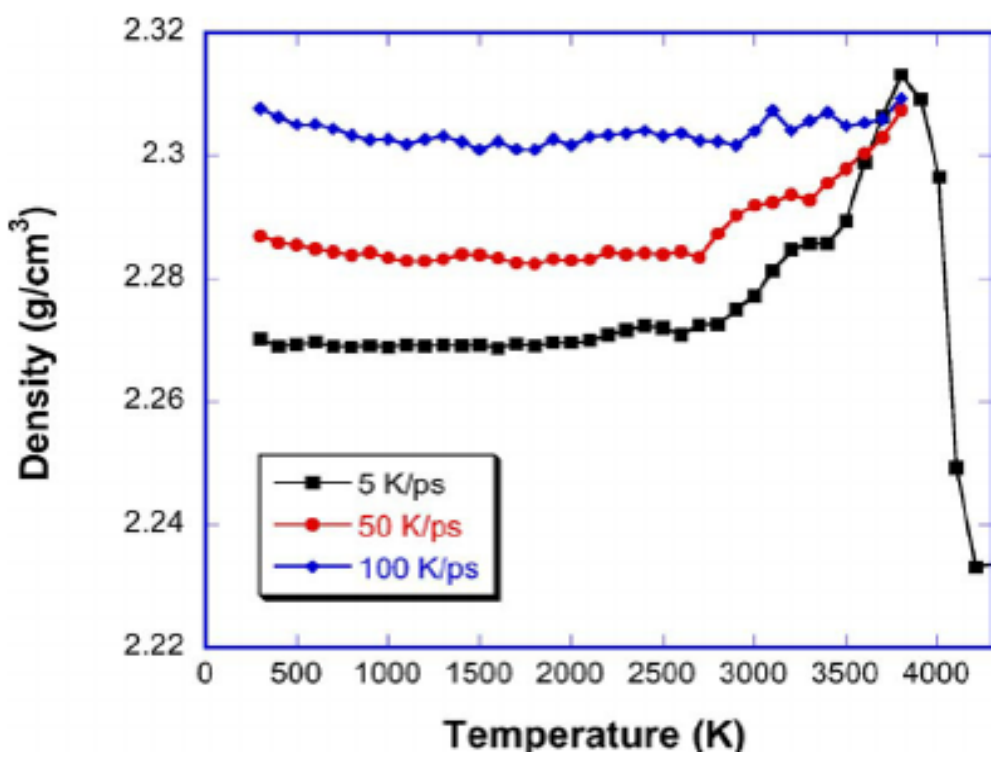

The pyknometer method of density analysis can be assumed to be well known. With careful work, results can be determined to the fourth decimal place [4]. The suspension method, by which the piece of glass being investigated is immersed in an original liquid of roughly the same density, makes possible an additional decimal place of accuracy [5]. Since the coefficient of expansion of the liquid is significantly greater than of the glass, by varying the temperature one can cause the piece of glass to be suspended at a constant height in the liquid column. Below chart is the example of that which shows the unexpected falling from density after some temperature. $\rho_{x}=\rho_{s}-0.0017\left(T_{x}-T_{s}\right)$

where, $\rho_{x}$ is the main density, $\rho_{s}$ is the density of standard glass and this is similar for $T$ which means temperature of the glass (The number 0.0017 is a temperature of density of the glass).

\subsection{Theory of Measurement}

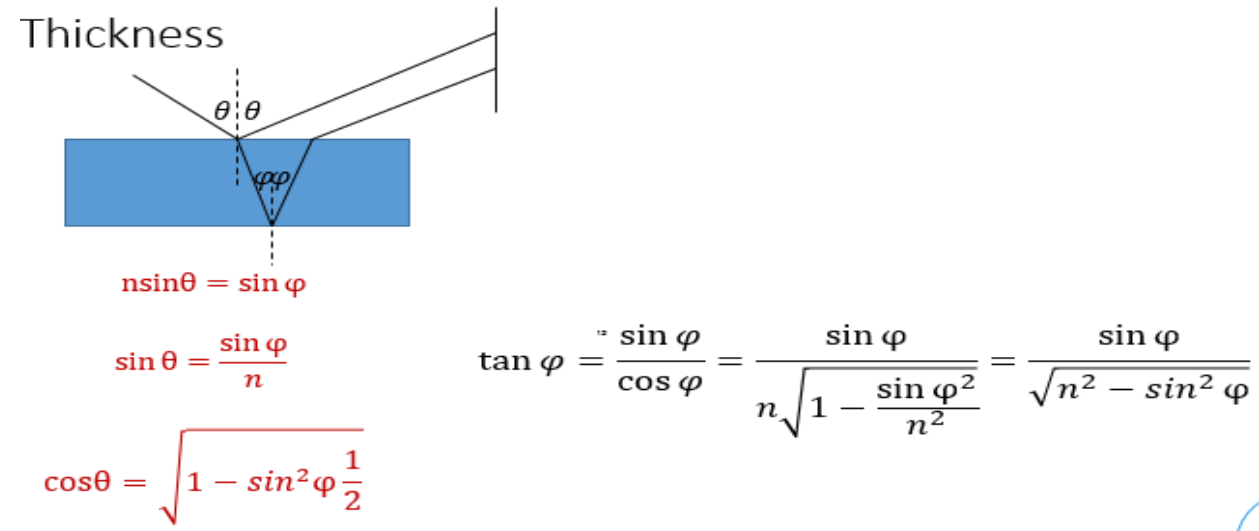

$\tan \varphi=\frac{2 d \tan \theta}{x}$ by snels and lambert law and the new theory which make from are manipulation on trigonometric [6].
The main variation are the main reflections angels $\theta$ and $\varphi$, then you earn thickness (d) with relation to them. 


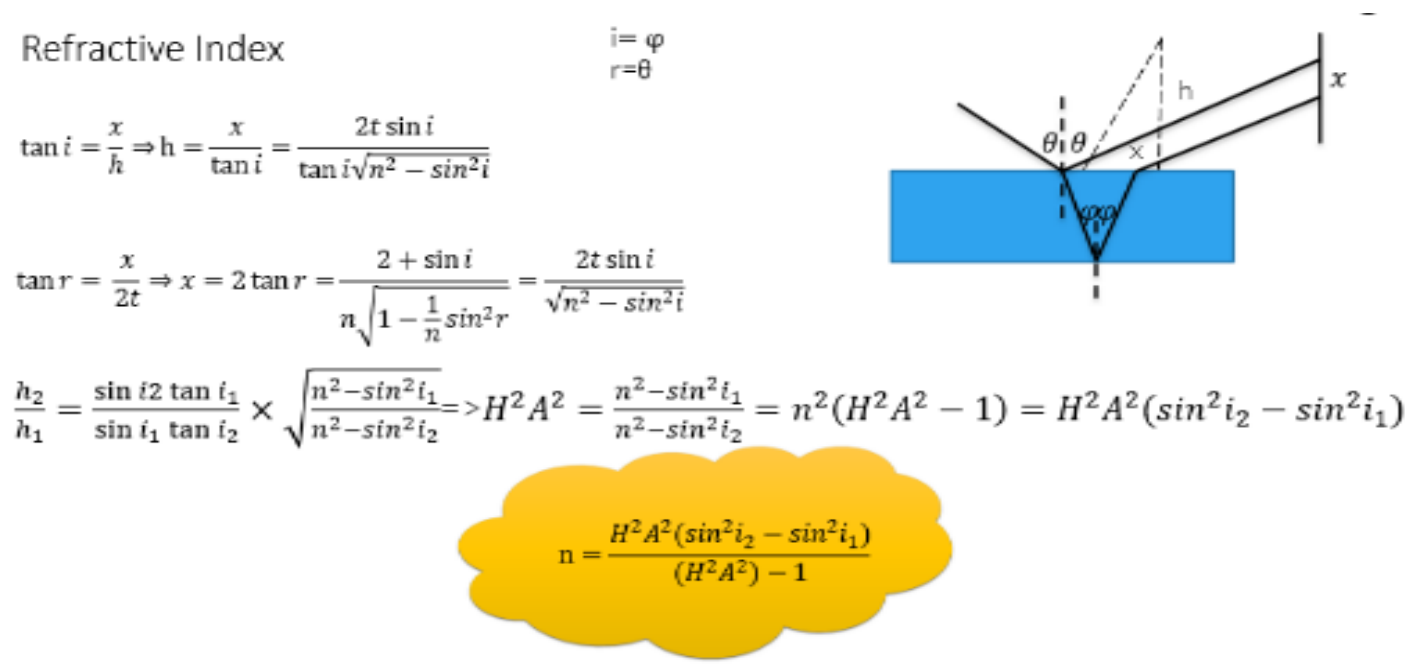

Here you can look at the refractive index theory, this the hardest part of theory because we do not know the amount of thickness and want to find both of them with device [7]. Solution is AI (Artificial intelligence), we thought Arduino to send the refractive index code after the thickness finding code. This theory consists of naming theory which is the way we usually solve our problems. Again, it begun with reflection angels and went through optical laws and gave us refractive index after naming theory.

\section{Where Can You Use It}

Glasses of course have being used in the field of Building \& Construction since many of us surrounded by sky scraper tower. In addition, air industry, the role of airplane windows is vitally important today, most of them have the property of impenetrability, not letting X-ray to pass them and have a light weight. These properties must be checked weekly or fortnightly but at present we still do not have any comfortable device to do them. An optical device may be used just in 2 second from onside of the airplane window. Some airplane companies are interested in that and we hope to gain the attention of huge air.

\section{References}

[1] Glass, Nature and Structure, Horst Scholze. 1996.

[2] Contactless online thickness measurement-alpha.ti (nokra.de).

[3] Dr. Holloway, G. 1985. The Physical Properties of Glass.

[4] Spengler, S., Munkes, D., and Sparschuh, G. 1997. Apparatus for making contactless measurements of the thickness of an object made of transparent material. US Patent 5636027 A (Jun. 3, 1997).

[5] Wilke, T., Witzmann, A., Fehr, R., Faderl, J., Schmittel, O., Schaefer, E.-W., and Fritsch, C. 2008. Method and apparatus for contactless optical measurement of the thickness of a hot glass body by optical dispersion. US Patent 7414740 B2 (Aug 19, 2008).

[6] Tole, W. R. 1990. Apparatus for determining the thickness of material. US Patent 4902902 A (Feb 20, 1990).

[7] Larina, T. V., Kutenkova, E. Y., Rakhimov, N., and Ushakov, O. K. Contactless glass sheet thickness meter. Russian Patent 2429447. 